\title{
Perspective
}

Actualité en histoire de l'art

1 | 2017

actualité en histoire de l'art

\section{La forêt de Fontainebleau : une nature monumentale, un monument naturel ?}

The Fontainebleau Forest: From Monumental Nature to Natural Monument?

Der Wald von Fontainebleau: monumentale Natur, natürliches Monument?

La foresta di Fontainebleau: dalla natura monumentale al monumento naturale?

El bosque de Fontainebleau: naturaleza monumental o monumento natural?

\section{Chantal Georgel}

\section{(Q) OpenEdition}

\section{Journals}

Édition électronique

URL : https://journals.openedition.org/perspective/7226

DOI : $10.4000 /$ perspective. 7226

ISSN : 2269-7721

Éditeur

Institut national d'histoire de l'art

Édition imprimée

Date de publication : 30 juin 2017

Pagination : 129-143

ISBN : 9782917902387

ISSN : 1777-7852

Référence électronique

Chantal Georgel, «La forêt de Fontainebleau : une nature monumentale, un monument naturel ? " Perspective [En ligne], 1 | 2017, mis en ligne le 31 décembre 2017, consulté le 16 janvier 2023. URL http://journals.openedition.org/perspective/7226 ; DOI : https://doi.org/10.4000/perspective.7226 


\section{La forêt de Fontainebleau : une nature monumentale, un monument naturel?}

Le 5 décembre 2016 débutaient en forêt de Fontainebleau, dans le chaos rocheux d'Apremont, proche de Barbizon, d'importants travaux d'abattage de centaines d'arbres ; cette coupe suscita, immanquablement, l'ire du quotidien écologiste Reporterre, lequel publiait le 9 février 2017 une tribune hostile intitulée " Massacre au bulldozer en forêt de Fontainebleau ", mais surtout un vrai étonnement : pourquoi couper des arbres sains, jeunes, vigoureux ? Que signifiait cette intervention? Au visiteur qui s'interrogeait, légitimement, l'Office National des Forêts (ONF) apportait une réponse claire, affichée sur un panneau installé à l'entrée du site. Ces travaux avaient pour objectif de " redessiner les paysages qui inspiraient autrefois les peintres ", de " mettre en valeur un patrimoine paysager ». Ce n'était donc ni un abattage économique, ni une coupe d'entretien, mais un travail de restauration, d'un fragment de nature, d'une nature pourtant naturellement vivante et vivace, situé dans le temps, ce temps étant celui " des peintres ». Cette gestion de la forêt prenant en considération son artialisation est une rare et belle idée, particulièrement adaptée au paysage d'exception qu'est la forêt de Fontainebleau (forêt de protection, Natura 2000, site classé, réserve de biosphère, réserve biologique, ZNIEFF), où très tôt s'est noué un dialogue fécond entre art et nature, qui la fit fréquenter par des centaines d'artistes, peintres, photographes puis cinéastes, la transformant en un véritable " atelier grandeur nature " (GEORGEL, 2007).

La réputation du lieu, portée par des noms prestigieux, Jean-Baptiste Camille Corot, Théodore Rousseau, Jean-François Millet et l'école dite de Barbizon, et même quelque peu par Frédéric Bazille, Auguste Renoir ou Claude Monet, est indéniable et justifie la prise en compte par l'ONF de ses paysages dans ses plans d'aménagement (FREYTET, 1996), au nom de l'attente des dix millions de visiteurs qui viennent chaque année randonner en forêt et visiter ses villages, dont le célèbre Barbizon. Pourtant, cette opération de restauration, comme toute opération de restauration d'un objet patrimonial, monument ou tableau... et ici paysage, pose de nombreuses questions, dont celle de la légitimité du choix de l'état à restaurer. Ce " temps des peintres ", quel est-il réellement, comment s'inscrit-il dans le temps long de la forêt ? De quelle réalité, de quelles images s'est-il inspiré ? Au final, de quel état de l'art, et donc de l'imaginaire (bien plus que du réel ?) du lieu, cette restauration est-elle l'aboutissement?

Ces questions émergent parfaitement d'une lettre écrite par Eugène Fromentin (18201876), alors jeune élève de Jean-Charles Reymond, le 28 juillet 1844, à sa mère, après avoir visité pour la première fois le pays de Fontainebleau, lettre curieuse et passionnante :

Chantal Georgel, conservateur général du patrimoine et conseiller scientifique à l'INHA, est membre du comité scientifique chargé par la ville de Fontainebleau d'élaborer le dossier d'inscription de la forêt de Fontainebleau au patrimoine mondial de l'Unesco. 
" Le pays, disait-il, est au niveau de ce qu'on peut imaginer de grand et de beau. J'en suis quelque peu étourdi ", tandis qu'à un ami (Paul Bataillard), il confiait le même jour : " Je ne connais encore qu'un coin de la forêt. Ce n'est pas au-dessus de ce que j'avais rêvé, mais $c^{\prime}$ est bien au niveau ${ }^{1}$. " Chaque mot de ces impressions résonne comme une interrogation : qu'avait-il pu imaginer ? Pourquoi cet étourdissement ? De quelles images, de quelles significations ce lieu avait-il été investi, qui justifient ces propos et ce que ce jeune artiste n'hésite pas à désigner du nom de rêve ?

Répondre à ces questions appelle une enquête approfondie. Il importe de quitter les sentiers balisés de l'histoire des peintres et de la peinture de paysage ${ }^{2}$ pour se tourner (d'abord) vers "l'objet rêvé ", la forêt de Fontainebleau, son histoire et surtout celle de ses représentations, mentales et/ou visuelles. Un long cheminement au travers de dizaines de textes (certains remontant au XVII ${ }^{\mathrm{e}}$ siècle, mais la plupart datant des années 1740-1840), à la rencontre de botanistes, géologues, poètes, dramaturges, romanciers, voyageurs, peintres aussi, permet alors de mettre au jour un paysage étonnant au regard d'aujourd'hui, un paysage imaginé sinon imaginaire si on le confronte à notre perception du lieu, mais cohérent mentalement, intellectuellement et visuellement, suffisamment pertinent aux yeux des artistes pour qu'ils n'aient pu échapper à sa magie, pour qu'ils l'aient investi et aient livré à la postérité des centaines d'œuvres, lesquelles, devenues "images ", mentales peut-être davantage que visuelles, devaient à leur tour à la fois créer et nourrir un imaginaire collectif, celui-là même auquel sont confrontés (à moins qu'ils n'en soient eux-mêmes porteurs) les aménageurs de la forêt, dont la réflexion paysagère ne saurait faire abstraction, tant elle ne peut faire abstraction des représentations culturelles et symboliques de l'objet à aménager.

\section{Un paysage construit}

L'appréciation de la beauté, de la valeur esthétique d'un paysage est toujours subjective et ancrée dans le temps. La forêt de Fontainebleau n'échappe pas à la règle. Comme toute forêt occidentale, elle fut longtemps absente du regard de l'homme qui, lorsqu'il la percevait, ne savait que s'en défier (HARRISON, 1994). Laissée dans l'indéfini, innommée. Charles Estienne recommandait encore en 1552 de l'éviter, dans La Guide des chemins de France, et si le Père Dan, premier historien du château, parvient à l'évoquer, en 1642, c'est parce que loin de ternir la gloire du château, elle la sublime : " c'est, dit-il, une merveille, de voir sortir la lumière des ténèbres et l'abondance de la stérilité ${ }^{3}$ ". Lentement, et laborieusement, cette forêt réussit dans le courant du XVII ${ }^{\text {e }}$ siècle, à être enfin qualifiée de quelque adjectif, vaste ou sauvage, voire belle, par la peu conformiste princesse Palatine qui, le 20 octobre 1694, se désolait auprès de sa " chère Louise " de devoir la quitter. Mais ce n'est pas avant le XVIII ${ }^{\mathrm{e}}$ siècle que ce milieu naturel devient un cadre mental offert à la spéculation intellectuelle et aux plaisirs ou aux tourments de la sensibilité.

La célèbre Description historique des chasteau, bourg et forêt de Fontainebleau publiée en $1731^{4}$ par l'abbé Guilbert, louée d'abondance par le Journal des savants (dès juillet 1731), permet à la forêt de Fontainebleau de faire irruption sur la scène parisienne à laquelle elle donne à " voir " ce " surprenant désert de Franchard ", auquel on accède après " une lieue et demi de chemin, à travers des montagnes escarpées, des sables arides et de monstrueux et brûlants cailloux [tandis que] des milliers de rochers entassés avec peine, et escarpés à l'envi, dérobent toute autre vue que la région céleste, et forment uniquement le plan, le dessin et les perspectives de cette solitude ». Plan, dessin, perspective, l'écriture de l'abbé Guilbert fait surgir sous les yeux un tableau du désert de Franchard curieusement similaire à celui peint par Jean-Baptiste Oudry en 1738, La Chasse au cerf dans les rochers de Franchard (fig. 1). 


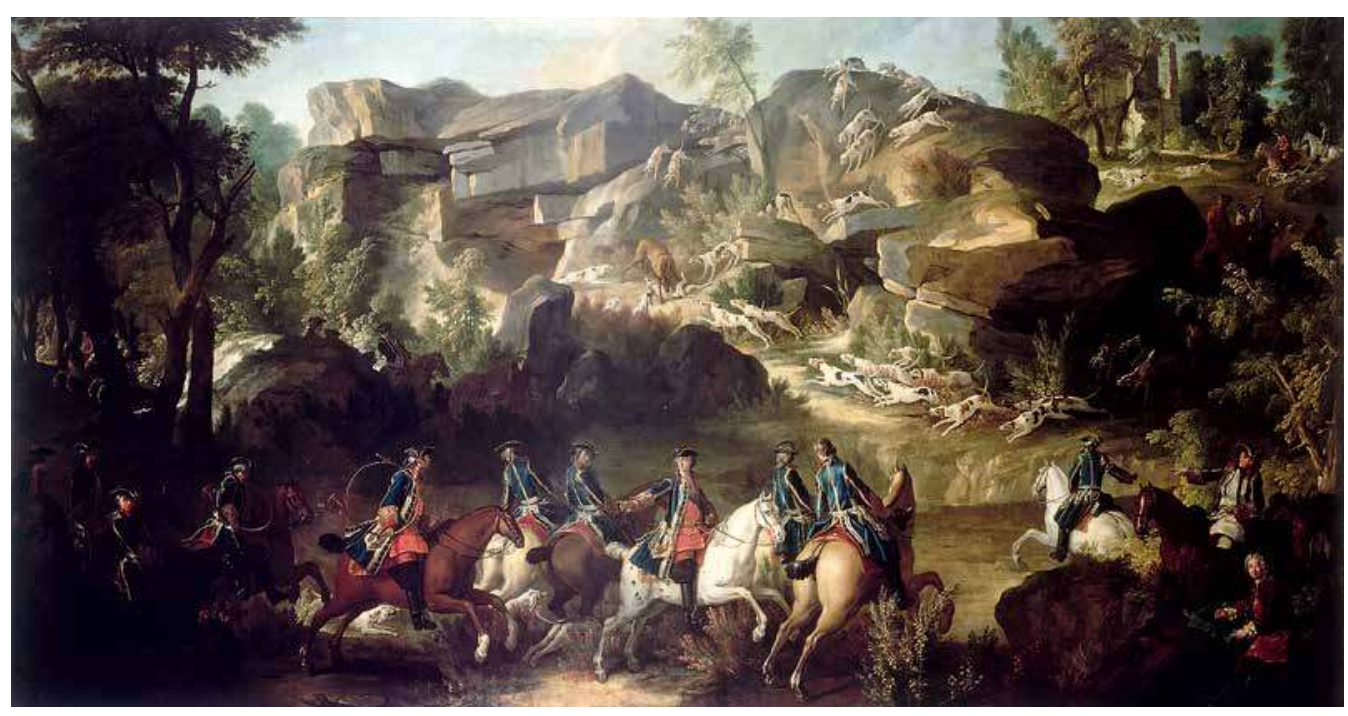

1. Jean-Baptiste Oudry, La Chasse au cerf dans les rochers de

Franchard, forêt de Fontainebleau, 1738,

Fontainebleau, château.

Le paysage domine l'ensemble. Le roi (qui nous fait face) et les officiers qui l'entourent au premier plan, dans une semi-pénombre, semblent comme arrêtés par une falaise de rochers d'où dévalent les chiens et qui barre un horizon sur lequel butte notre regard. C'est à peine si l'on songe à l'en détourner pour apercevoir, fuyant sur la droite, une petite clairière nimbée de la poésie des ruines qu'elle conserve, qui sont celles de l'ermitage de Franchard détruit sur ordre du roi le 20 février 1717. Ces deux regards, contemporains l'un de l'autre, sont comme les premiers symptômes d'une sorte de "fièvre bellifontaine " qui devait s'emparer des esprits quelques décennies plus tard, à la fin du XVIII siècle et dans les premières années du XIX ${ }^{e}$ siècle, portée en premier lieu par les mots de l'écrivain et du poète, dont Étienne de Senancour et René-Richard Castel, lesquels allaient offrir à la forêt les fondements de son identité.

En 1785, à l'âge de 14 ans, Senancour (1770-1846) découvre la forêt de Fontainebleau, où il retournera à plusieurs reprises, en 1786, 1788, entre 1790 et 1794 et probablement une dernière fois vers 1810. De ces séjours, il tire un roman autobiographique, Obermann (publié en 1804, réédité en 1833 par George Sand et Sainte-Beuve) qui livre, en une série de lettres fictives, ses impressions de la forêt, impressions ineffaçables de l'adolescence, empreintes d'un sentiment profond de solitude, d'abandon : " je trouvai une solitude assez austère comme l'abandon que je cherche ", écrit-il en sa lettre XVIII. Solitude, abandon, liberté, ces mots ont pris possession de la forêt de Fontainebleau, bientôt accompagnés de ceux du poète RenéRichard Castel (1758-1832), émule improbable de Chateaubriand. Il est le premier (parmi les bellifontains) à employer, dans son poème intitulé La Forêt de Fontainebleau (publié chez Deterville en 1805), le mot qui va célébrer les noces de cette forêt avec toute une génération d'artistes : "romantique ". Après avoir décrit cette forêt " coupée en tous sens par de grandes chaînes de rochers " et où l'on trouve "ici des terrains couverts des plus beaux bois, là d'arides sablons où l'herbe même refuse de croître ", et là " un océan de sable étincelant de feux ", il ajoute en effet que l'on arrive enfin au " lieu le plus romantique " : " la solitude de Franchard [...] une gorge profonde où l'œil des deux côtés ne voit que rochers monstrueux ». Montagnes escarpées, sables brûlants, gorges profondes, rochers monstrueux : tels sont les paysages construits par les hommes de plume. Ces mots, qui dessinent de véritables tableaux, trouvent un écho chez le voyageur qui, depuis la route le conduisant vers Lyon, Genève, la Suisse ou l'Italie, est persuadé (ou se persuade) d'avoir sous les yeux « un diminutif 
2. Eugène Cuvelier, Sables de Macherin, 14 octobre 1863 Paris, $\mathrm{BnF}$ département des Estampes et de la Photographie, FOL-EO-224 (2)

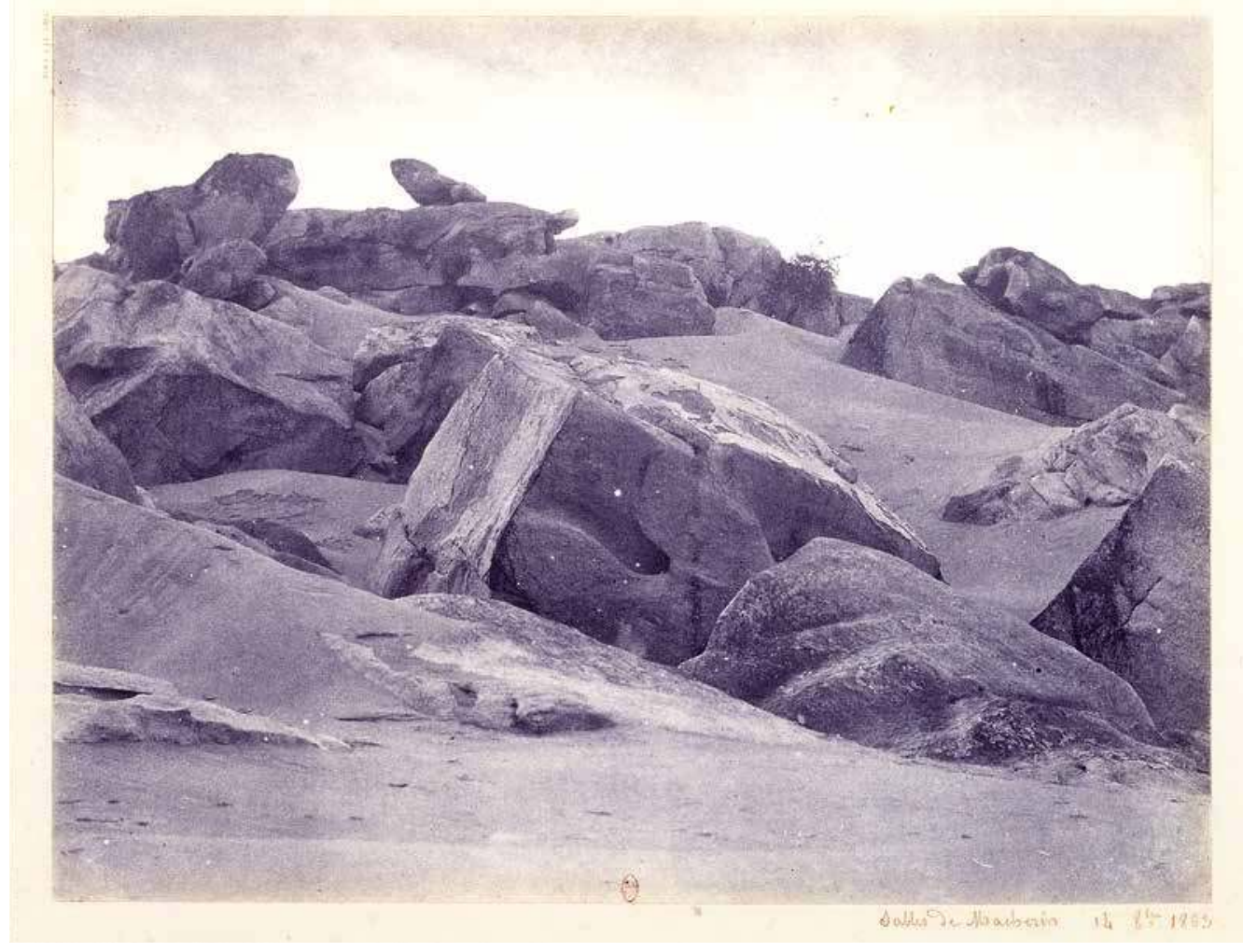

des Alpes " ", " une idée des beaux paysages de la Suisse ${ }^{6}$ ", tandis que les hommes de science, Cuvier et Brongniart, comparent les bancs de grès dénudés et chaotiques à un " grand nombre de lieux de l'Allemagne, de la Suisse, de l'Italie, qui présentent des terrains analogues " ". Une vision confortée par les études des naturalistes : "On y trouve un grand nombre de plantes qui lui sont particulières et dont plusieurs mêmes ne se trouvent que dans les Alpes ", explique ainsi, en 1824, Alexis Donnet dans sa Description des environs de Paris ${ }^{8}$, idée que l'on retrouve chez tous ses contemporains, parmi lesquels Jean-Antoine Dulaure dont la Nouvelle description des environs de Paris, contenant les détails historiques et descriptifs des maisons royales, des villes, bourgs, villages, châteaux, etc...remarquables, constamment rééditée de 1786 à 1853 , fait d'autant plus autorité qu'elle ne cesse d'être copiée, répétée.

En quelques années, la forêt de Fontainebleau était devenue, sous l'effet secondaire de la découverte de la montagne et de l'invention du Mont-Blanc, un paysage échappant à toute raison : qu'est-ce en effet que ces montagnes escarpées et ces gorges profondes que l'on ne cesse de vanter jusque tard dans le siècle (fig. 2) ? Le point culminant de la forêt est à 136 mètres, au Cuvier-Chatillon!

Une forêt qui ressemble aux Alpes, à la Suisse, " affreusement belle " (chacun répète le mot de Béranger), une forêt où règne une si grande variété que l'on y peut voir montagnes, roches, sables et arbres de tous âges, la forêt de Fontainebleau ainsi perçue et vécue se révélait propice à toutes les évocations, ce que confirmaient Senancour (dans le Mercure de France en janvier 1812) qui y voyait « quelque chose des formes bouleversées de la Finlande, et de l'aridité des monts de la Nubie ", ou ce jeune voyageur évoquant en 1835 à son propos les "forêts de l'Amérique ${ }^{9}$ ", propos repris plus tard, en 1867, par Frédéric Thomas Graindorge : 
« Est-il possible, s'exclame-t-il, qu'il y ait auprès de Paris une forêt pareille ? Tous mes souvenirs d'Amérique se sont réveillés ${ }^{10}$. " À Théophile Gautier elle offrait l'illusion de se trouver à "Smyrne, avec ses hauts cyprès se profilant sur l'émail bleu du ciel ${ }^{11}$ ", tandis que d'autres encore y voyaient le Sahara ${ }^{12}$, la savane ou bien une steppe couverte de bruyères, quand ils n'y retrouvaient pas les sensations que laisse une mer - " une mer verte " disait Michelet -, un océan, ses plages et ses dunes. "Ce qui fait la forêt de Fontainebleau unique, " concluait admirablement Émile Verhaeren dans L'Art moderne, le 10 novembre 1889, " c'est sa variété. Elle résume toutes les forêts : les héraldiques, les frustes, les farouches, les jolies, les terribles, celles du nord avec leurs sapins, celles du centre avec leurs bouleaux et leurs chênes, les forêts du rêve et de la vie, toutes" ".

Une forêt qui pouvait tenir lieu de tout, voilà qui devait intéresser les artistes.

\section{Un paysage investi}

François-Hippolyte Paillet, dans sa Description physique de la forêt de Fontainebleau, publiée sous l'Empire, fut le premier à convier les peintres à Fontainebleau. "Les paysagistes, écrit-il, n'ont rien de mieux à faire que d'y venir passer quelque temps pour étudier la nature. Des arbres et des rochers de toutes sortes de formes leur fourniront abondamment de quoi exercer leurs crayons ou leurs pinceaux, soit qu'ils s'attachent à quelqu'un de ces objets en particulier, soit qu'ils en groupent plusieurs ensemble ${ }^{13}$. " Charles Remard, dans son guide de 1820, et Dulaure, en 1825 , renouvellent cette invite à venir étudier ces " rochers tout-à-fait nus ou garnis quelquefois de hêtres ou de chênes assez beaux, et surtout de vieux bouleaux dont les tons blancs se détachent agréablement sur le gris sombre et bleuâtre des rochers ${ }^{14}$ ".

La fin du XVIII ${ }^{e}$ siècle et les premières années du XIX furent le temps où la forêt de Fontainebleau devint un paysage, et ce fut le temps des premières incursions de peintres en ce paysage. Jean-Joseph Bidauld, Georges Michel, Jules-Etna Michallon, ou encore LouisÉtienne Watelet, Jean-Louis Demarne, Hyacinthe Dunouy, Nicolas-Fréderic Knyp furent les premiers à fréquenter les sous-bois de Fontainebleau, pour des ouvres parfois classiques, bientôt suivis par de nombreux confrères, tels Richard Parkes Bonington, Jacques Raymond Brascassat, Alexandre Desgoffe ou Jules Coignet et Xavier Leprince, dont les petits tableaux intitulés Peintres sur le motif (1825, Barbizon, musée départemental de l'École de Barbizon) et Halte des peintres à Fontainebleau (vers 1820, Beauvais, musée de l'Oise) témoignent de la naissance d'une pratique, tout comme l'Artiste passant dans un chaos de rochers à Fontainebleau (fig. 3) de Corot. Il y avait fait ses premières études d'arbre, le 8 octobre 1822 (Détails de tronc d'arbre en forêt, Barbizon, musée départemental de l'École de Barbizon), avant d'y revenir régulièrement, tandis que Théodore Rousseau y peint sa première Étude de rochers en 1829 (Strasbourg, musée des Beaux-Arts).

La vente de l'atelier de Michallon (12 études de la forêt de Fontainebleau) le 26 décembre 1822, la parution d'un ensemble de recueil et d'albums lithographiques incluant des Vues de Fontainebleau ${ }^{15}$, la publicité insensée faite à cette forêt par le colonel Juncker qui pense y avoir découvert en 1823 un fossile humain, témoin du Déluge ${ }^{16}$ (et exposé boulevard des Capucines), une

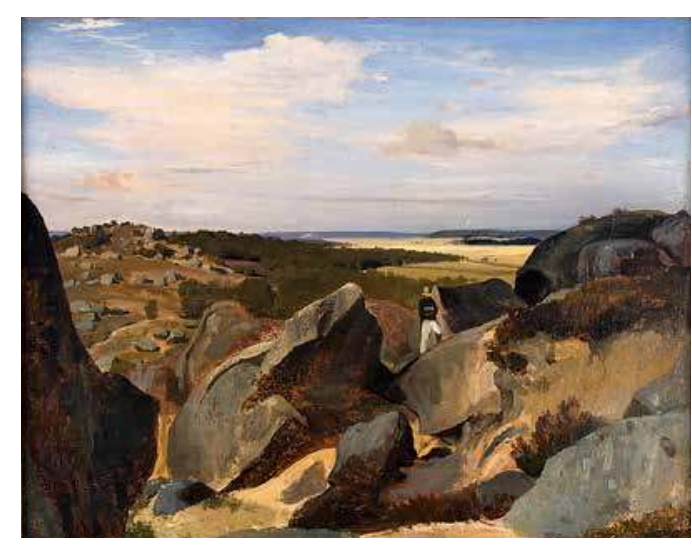

3. Jean-Baptiste Camille Corot, Un Artiste passant dans un chaos de rochers, vers 1830 Neuchâtel, musée d'Art et d'Histoire. 
4. Jean-François Hue, Vue prise dans la forêt de Fontainebleau, 1783 ,

Fontainebleau, château.
5. Jean-Léon Gérôme,

Panthère noire sur un rocher, 1851, Boston, Museum of Fine Arts.

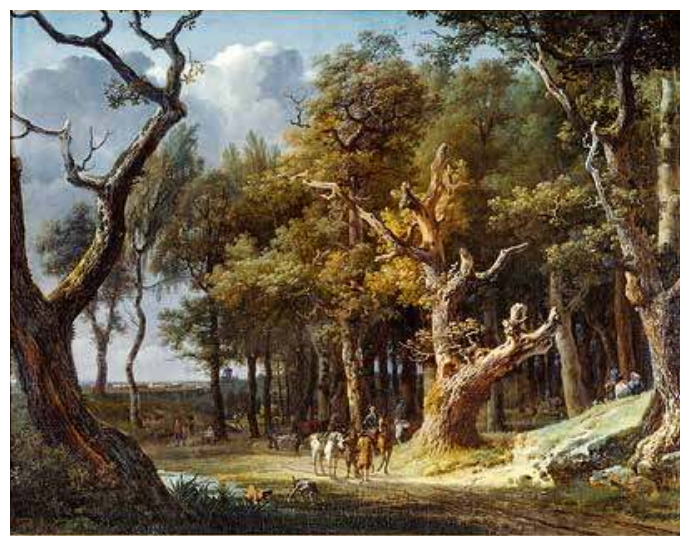

constante célébration des beautés du lieu par de grands noms de la littérature tels George Sand et Alfred de Musset, ou François-René de Chateaubriand, la diffusion de reportages illustrés dans Le Magasin pittoresque ou L'Illustration (véritables machines à fabriquer de l'imaginaire) et, enfin, le bouche à oreille des ateliers... amènent sous la monarchie de Juillet de telles foules d'artistes, peintres ou photographes, qu'il devient impossible de les nommer, mais les chiffres font sens.

On ne sait combien de peintres exactement sont venus travailler en forêt de Fontainebleau, mais le nombre d'artistes ayant présenté au Salon au moins une ouvre bellifontaine (ce qui exclut les vues de forêt, arbres et roches qui ne disent leur nom) est un indicateur signifiant : 557 peintres (de plusieurs nationalités) ont ainsi exposé 1258 œuvres indéniablement bellifontaines au Salon, de 1781 (date à laquelle est exposé la Vue prise dans la forêt de Fontainebleau de Jean-François Hue [fig. 4]) à 1879, aube de la Troisième République, et apogée de la peinture «bellifontaine ». Pendant plusieurs décennies, chaque Salon donne à voir entre dix et trente peintures inspirées de la forêt de Fontainebleau, et jusqu'à soixante-cinq (Salon de 1877). Ces chiffres disent combien les peintres, suivis par les pionniers de la photographie (Gustave Le Gray, Eugène Cuvelier ; CHAlle, 1991), ont été sensibles à cette image de la forêt de Fontainebleau telle qu'elle fut inventée, construite et amplement diffusée dans les premières décennies du siècle. Cette armada a su réfléchir à la fois à la signification profonde de ce lieu renvoyant perpétuellement

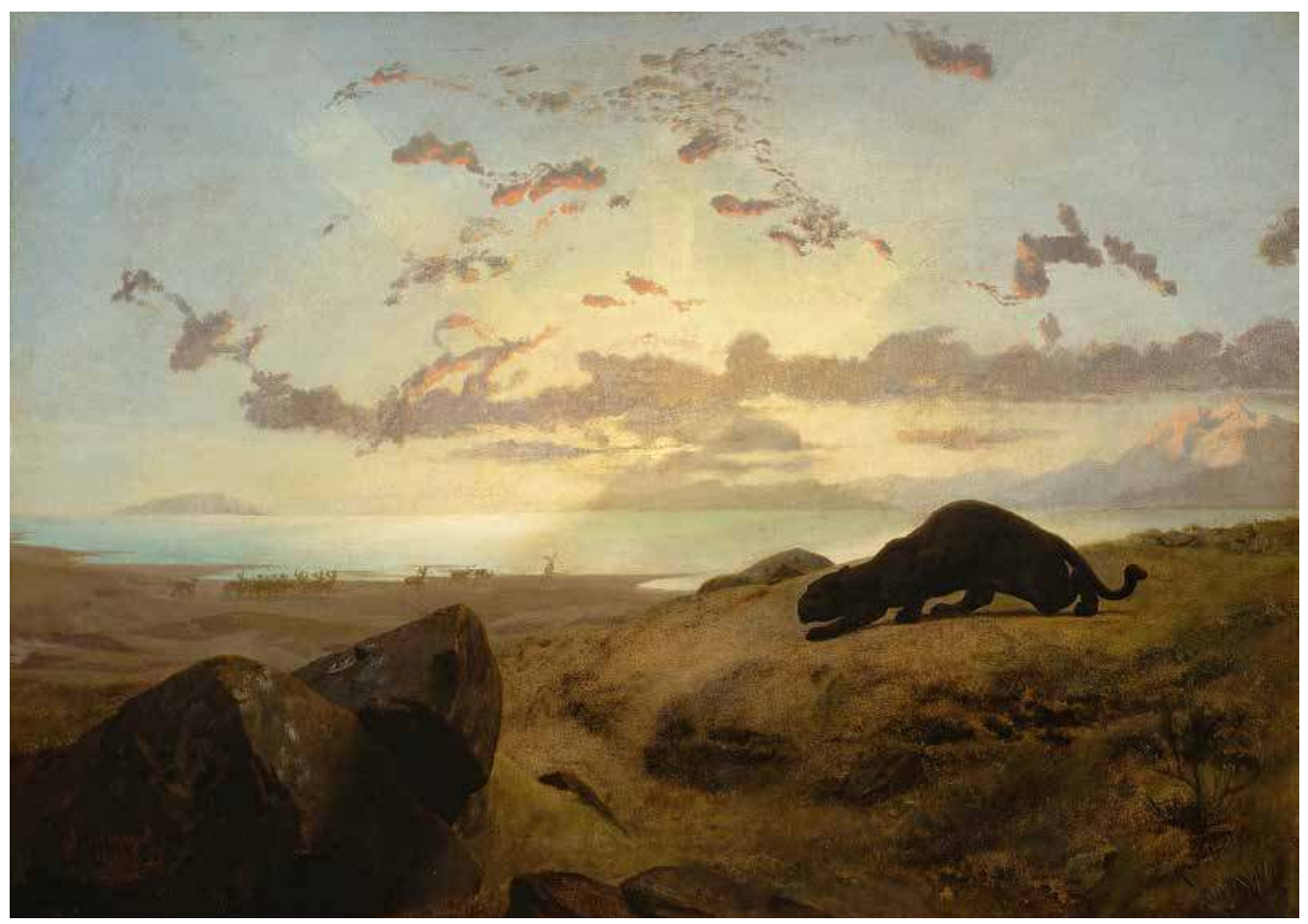


à d'autres lieux, invitant constamment à ce que Théodore Rousseau appelle joliment une "géographie comparée $\mathrm{e}^{17}$ ", et au travail de l'imagination à mettre en œuvre par l'artiste. En font foi les multiples forêts de Fontainebleau abritant tigres et panthères (fig. 5), légendes alsaciennes ou slovaques, et autres scènes orientales, avant que les premiers expérimentateurs du cinématographe naissant n'y introduisent Jésus Christ au mont des Oliviers (Jasset, La Passion $d u$ Christ, 1905 ; Zucca, La Vie et la Passion de notre seigneur Jésus Christ, 1906), et bon nombre de reconstitutions historiques (Capellani, Le Courrier de Lyon, 1905 ; Denola, Bonaparte et Pichegru, 1909; Andréani, Le Siège de Calais, 1910...) ! Près de 500 films ont été tournés à ce jour en forêt de Fontainebleau (JELOT-BLANC, 2008).

Cette forêt a donc su « tenir lieu de tout " mais il n'est pas interdit de se demander, avec Jean Borie (qui a érigé la forêt de Fontainebleau en objet littéraire), si ces foules
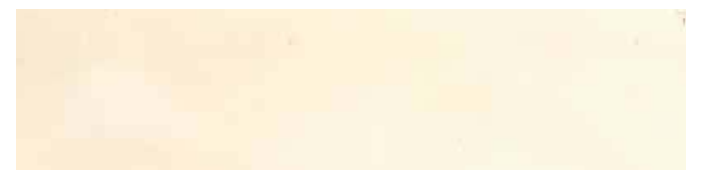

(1)
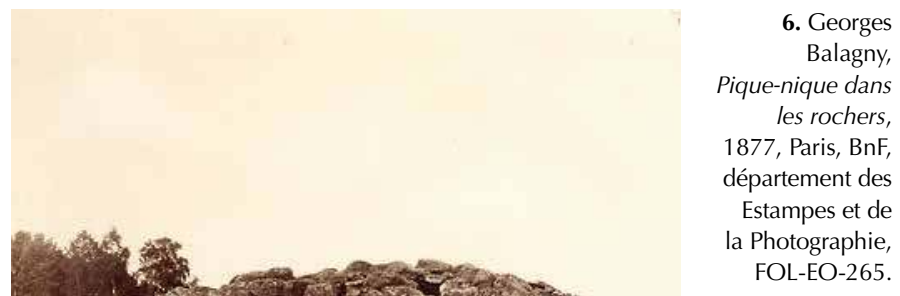

Georges ique dans rochers,

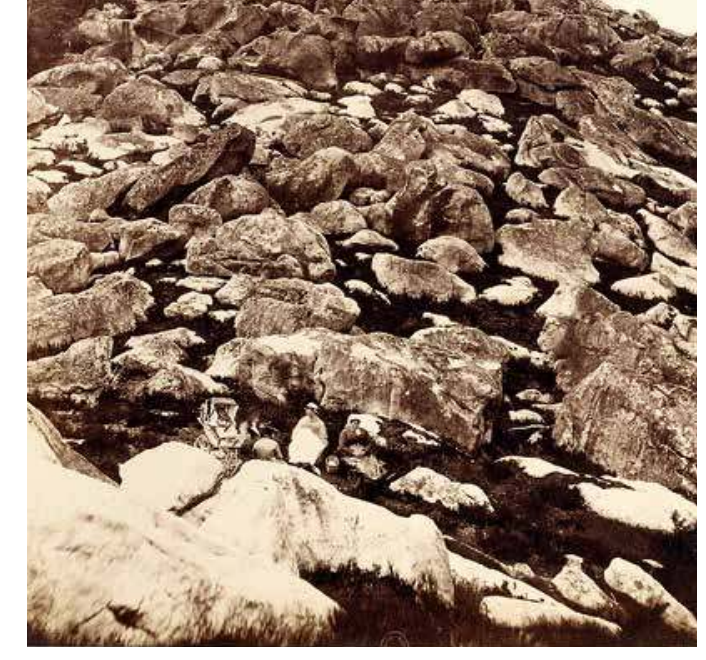
d'artistes ne seraient pas venues à Fontaine-

bleau aussi, avant tout, et plus prosaïquement, parce qu'ils y trouvaient " un ouvrage de référence, un peu court mais pratique, facile à consulter, toujours disponible sous la main [...], une sorte de Petit Larousse de la peinture " (BORIE, 2003) ?

Une analyse chronologique des ouvres présentées au Salon depuis 1781 nous éclaire sur les choix des peintres et leur conformité, ou non, à l'imaginaire primordial de Fontainebleau, cette montagne de roches, ce désert de sables.

Il est révélateur que le premier lieu cité, en 1817, soit La Croix de St-Héran, point d'entrée de Fontainebleau, au carrefour de la ville et de la forêt, et qu'il le soit avec une mauvaise orthographe (la Croix de Saint-Hérem). Ainsi débute l'apprivoisement du lieu, sa lente pénétration. Peu après, dès 1824, apparaissent les mentions de " roches ", et de " rochers ", que l'on étudie et peint dans la Gorge aux loups puis au Nid de l'aigle et au Bas-Bréau. Le discours analogique qui assimile (faute de savoir en exprimer autrement le curieux et la beauté ?) les grès de Fontainebleau aux montagnes des Alpes, auxquelles, il est vrai, les peintres et les graveurs avaient attribué lors de leur invention au XVIII ${ }^{\mathrm{e}}$ siècle les traits caractéristiques et presque canoniques d'un ensemble de masses rocheuses, leur vaut une plus-value esthétique incontestée, à laquelle les photographes furent particulièrement sensibles, les techniques de la photographie, la contre-plongée en particulier, leur permettant de donner à voir le chaos rocheux dans sa dimension alpine (fig. 6) !

L'arbre, les arbres, autres joyaux de cette forêt " antique " apparaissent en 1833. "Chênes " et " hêtres " se partagent les choix du peintre, et du photographe (fig. 7), avant que le bouleau ne vienne solliciter leur regard (vers 1850 ; fig 8), et ils demeurent anonymes avant de faire place au " Chêne Charlemagne ", au "Sully ", au " Henri IV » ou au « Rageur ", des arbres remarquables « consacrés par une histoire et qu'une collectivité humaine reconnaît comme élément de son patrimoine " (Robert Bourdu, dans BOURDU, JOUSSAUME, VIARD, 1988) 


\section{Anonyme} Grands Chênes, forêt de Fontainebleau, daguerréotype, vers 1840 , Paris, musée d'Orsay.

8. Eugène Cuvelier, L'Orage, forêt de Fontainebleau, vers 1860 , Paris, musée d'Orsay.

9. Antoine-Louis Barye, Le Rageur, Fontainebleau, collection municipale.
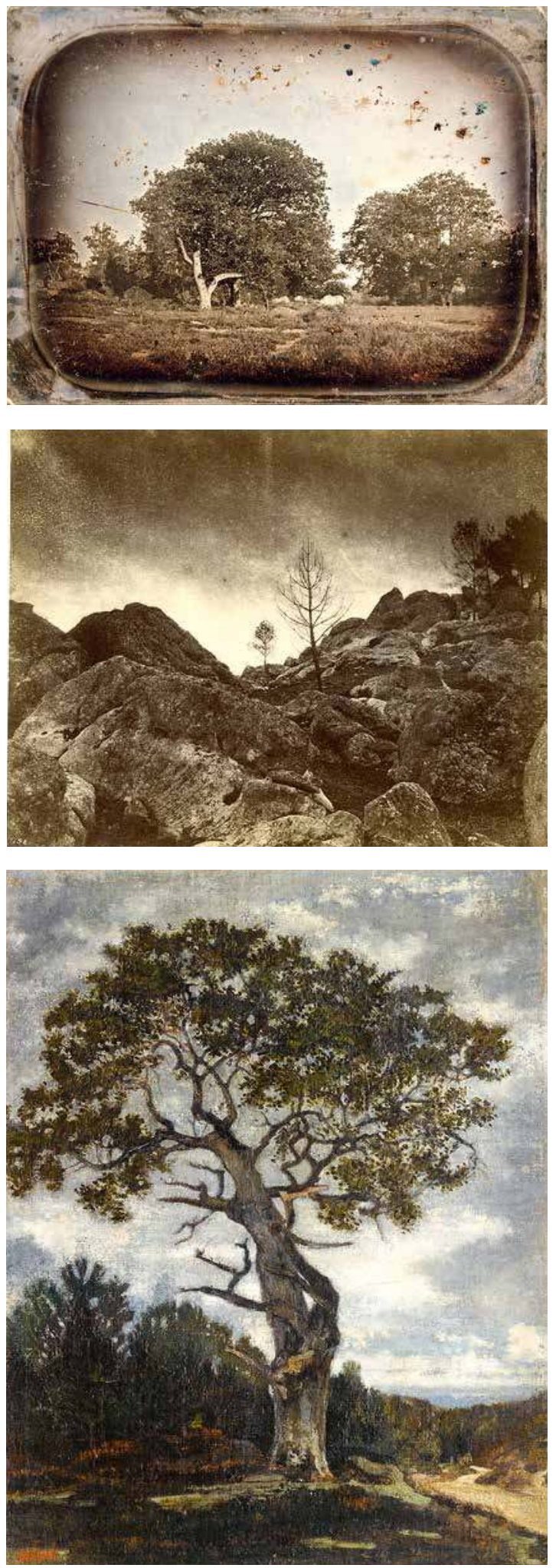

et célébrés dans toute la presse parisienne (fig. 9). L'évolution s'est faite parce que l'arbre a changé de statut pictural, passant au fil des ans du statut d'objet d'étude à celui de paysage en soi, offrant d'infinis jeux de matières, de couleurs, d'ombres et de lumières et parce plus généralement la quête du détail, du fragment, a fait place à celle du " motif " et très vite en forêt de Fontainebleau à celle du site, de plus en plus précisément situé.

Les Vues de la forêt, clairières, échappées, panoramas, accompagnent les études d'arbres et de roches durant tout le début du XIX ${ }^{\mathrm{e}}$ siècle. Recomposés en atelier, à partir d'études prises "sur nature ", ces paysages imprégnés de l'enseignement des Classiques offrent la satisfaction d'un univers vaste, infini mais dominé. Les Vues prises en forêt de Fontainebleau le sont depuis les "sommets", depuis les hauteurs de la Solle, qui forment corniche au-dessus de la vallée du même nom (Mme Sarrazin de Belmont, Vue prise des hauteurs de la Solle, 1834 ; Lanoue, Vue prise des hauteurs de la vallée de la Solle, forêt de Fontainebleau, $1836 \ldots$ ) ou depuis le promontoire du Calvaire, qui embrasse Fontainebleau et Avon (Mme Brice, Vue prise au Calvaire, forêt de Fontainebleau, 1835). Jean-Louis Gintrac (1808-1886) est le premier à présenter au Salon un Intérieur de forêt, en 1831, soulignant ainsi un mouvement de pénétration qui triomphe peu à peu. Pendant les premières années de la monarchie de Juillet, la vallée de la Solle, le mont Ussy et son Nid de l'Aigle, le mont Saint-Père, le Calvaire sont les lieux favoris des peintres (Antoine-Félix Boisselier, Au Calvaire en forêt de Fontainebleau, 1825, Senlis, musée $\mathrm{d}^{\prime}$ Art et d'Archéologie). Est-ce un hasard ? Ce sont ceux qu'Étienne Jamin dans son Guide de Fontainebleau publié en 1834 signalait comme étant " les principaux points de vue à visiter " : les rochers et la vallée de la Solle étant «sans contredit la plus belle situation pittoresque de la forêt ", il convenait cependant de s'arrêter au « carrefour de Bellevue, le long de la 
plaine de Chailly ", de " jeter un œil sur les gorges d'Apremont et de Franchard, à gauche de la route de Paris ", et de pousser "jusqu'au mont Aigu, à la droite de la route d'Orléans " jusqu'au « Haut Mont et la Gorge-aux-Loups, à la gauche de celle de Nemours ». Est-ce encore un hasard si en 1835, l'année suivant la publication de cette notice, Charles Claude Bachelier et Louis Cabat exposent chacun une Vue de la Gorge-aux-Loups, premières vues de ce site, l'un des plus fréquentés et les plus peints durant tout le XIX siècle, tandis que Philippe Bourgeois offre au Bas-Bréau sa première représentation officielle ? L'année suivante, les paysages du Déluge, puis ceux des monts Girard, du mont Chauvet, de la Mare-aux-Évées, de la porte aux Vaches, ainsi que ceux du rocher Canon, du rocher Fourceau, et du Jean de Paris font leur apparition au Salon, augurant de longues séries, et d'une diversification de plus en plus prononcée des sites représentés. On travaille désormais non plus seulement aux alentours de Fontainebleau, mais aussi aux portes de Bourron et de Marlotte, près de Chailly et de son hameau Barbizon. Claude-François Denecourt n'est certainement pas pour rien dans cette évolution. Cet ancien soldat de l'Empire devenu républicain, installé à Fontainebleau depuis 1832, après être tombé littéralement amoureux de la forêt, eut l'idée de faire profiter les visiteurs de son expérience intime du lieu. Intuitif, il avait aussi senti (ou compris) combien le tourisme était appelé à se développer et, plutôt avisé en affaires, s'était lancé dès 1839 dans une longue série de publications de guides, itinéraires, conseils, cartes de la forêt destinés à en faciliter la visite $^{18}$ (POLTON, [1985] 1994 et 2011). Les sentiers qu'il crée à partir de 1842, leur balisage à partir de 1847, favorisent évidemment de nouvelles pratiques de la forêt, en particulier par les peintres, auxquels ses guides s'adressent expressément. Son Guide du voyageur et de l'artiste à Fontainebleau de 1850 (fig. 10) fait état des "sites les plus recherchés des peintres", soit les " arbres séculaires les plus remarquables", ainsi que les « Rochers et points de vue ». Il désigne formellement au pinceau de chacun, par exemple " Au bas Bréau : 25 chênes et 4 hêtres; aux gorges d'Apremont : 15 chênes et 3 hêtres; à la Gorge-aux-Loups et au fort de Marlotte : 18 chênes, 3 charmes et 4 hêtres; Au mont Ussy et Nid de l'Aigle : 28 chênes et 6 hêtres, au rocher Cuvier : 3 chênes, 2 hêtres, 4 bouleaux et 10 genévriers. " Quant aux « endroits où l'on peut dessiner ou peindre de beaux rochers et de belles vues, [ils] se trouvent au mont Ussy, au sentier des Fées, au sentier des DeuxSoeurs, au mont Chauvet, aux gorges de la Solle... » Dénecourt réitère ses observations dans le Guide historique et descriptif qu'il publie en 1856, "ouvrage enrichi de cartes et de planches et le plus complet qui ait paru sur Fontainebleau ", et dresse en 1860 une nomenclature des vingt-quatre "points les plus remarquables et sites imposants qui font de Fontainebleau « un musée national donné par Dieu même à la France comme le modèle des paysages terrestres " et " le rendez-vous le plus fréquenté par les artistes et les touristes européens ${ }^{19}$.

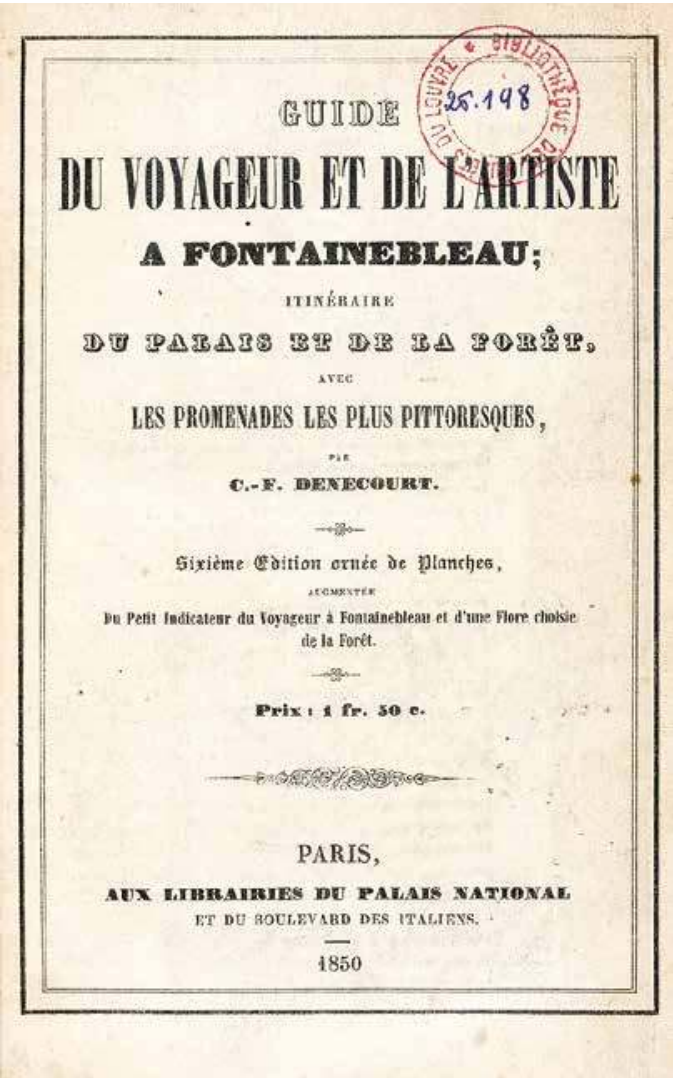


Cette forêt faisait l'objet d'un consensus national. En 1875, le journaliste et écrivain Jules Levallois publiait Mémoires d'une forêt. Fontainebleau (LEVALLOIS, 1875), un texte qui élevait le site au rang de "monument national " et lui conférait une forme d'humanité, tandis qu'en 1903 paraissait le Dictionnaire historique et artistique de la forêt de Fontainebleau, rédigé par Félix Herbet, homme de grande érudition épris de la forêt (HERBET, 1903), un « monument " au service de la forêt, une "forêt monument ", à protéger, à restaurer comme tout monument.

\section{Un paysage surinvesti ?}

Le paysage bellifontain s'étant installé dans l'œil des artistes et de leur public, la forêt de Fontainebleau fut très tôt saisie comme un paysage à préserver, au nom d'abord de ses arbres, les " arbres remarquables " étant perçus dès les premières décennies du XIX siècle et jusqu'à aujourd'hui comme des êtres à part entière, libres, porteurs d'histoire, et qui doivent nous survivre (Paul Arnould, dans CORVOL, ARNOULD, HOTYAT, 1997). Théodore Rousseau fut le premier à batailler pour la défense des arbres et leur protection, aussi bien contre les coupes des forestiers que contre les aménagements de Denecourt (fig. 11). Peut-être est-ce lui qui suscita l'article de Jules Janin, publié par L'artiste en 1839 ( La Forêt de Fontainebleau. Dévastations »), première sonnette d'alarme tirée dans la presse à l'encontre de quiconque viendrait perturber le paysage des peintres. Peut-être fut-il aussi à l'origine de la campagne d'opinion menée par les journalistes et les hommes de l'art en 1850, toujours contre les abattages d'arbres, opérés selon eux dans un but exclusivement économique. "Admirons sans réserve et comme il convient les belles choses entreprises sous la République : la restauration du Louvre et du Salon carré, la restauration du salon d'Apollon, les derniers achats faits en Hollande, et une foule d'autres travaux confiés à des artistes aussi habiles qu'intelligents ; mais que tout cela ne soit pas payé avec notre forêt ", plaidait L'Illustration du 26 octobre 1850, qui proposait en conclusion d'en confier la gestion à la Commission des monuments historiques. Sus aux coupes d'arbres, sus aux plantations nouvelles, tel était le mot d'ordre lancé par les artistes ! C'est alors que Théodore Rousseau entreprit, en 1852, d'écrire au duc de Morny, au nom "de tous les artistes qui peignent la forêt ", pour attirer son attention sur les « dévastations qui se commettent par l'administration elle-même dans la forêt de Fontainebleau ". Après avoir rappelé l'ancienneté de cette forêt " seul souvenir vivant qui nous reste des temps héroïques de la patrie depuis Charlemagne », le fait qu'elle ait " donné à l'art des artistes comme Lantara, Michalon [sic], Bruandet, Bertin, Th. Rousseau, Cabat et Troyon ",

11. Théodore Rousseau, Le Chêne brisé en forêt de Fontainebleau, Paris, musée du Louvre.

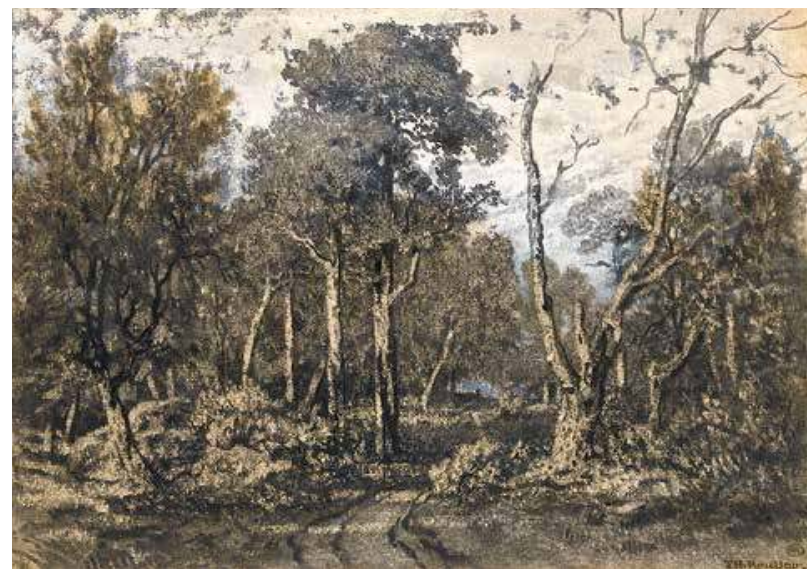
et que "pour les artistes qui étudient la nature elle est ce qu'est [sic] pour d'autres les modèles qui nous ont été laissés par Michelange, Raphaël, Corrège, Rembrandt et tous les grands maîtres des tems [sic] passés ", il supplie " que les lieux qui sont pour les artistes des sujets d'études, des modèles reconnus de compositions et de tableaux, soient mis hors l'atteinte de l'administration forestière qui les gère mal, et de l'homme absurde qui les exploite ${ }^{20} »$. Ces prises de position, ces pressions, aboutissent 


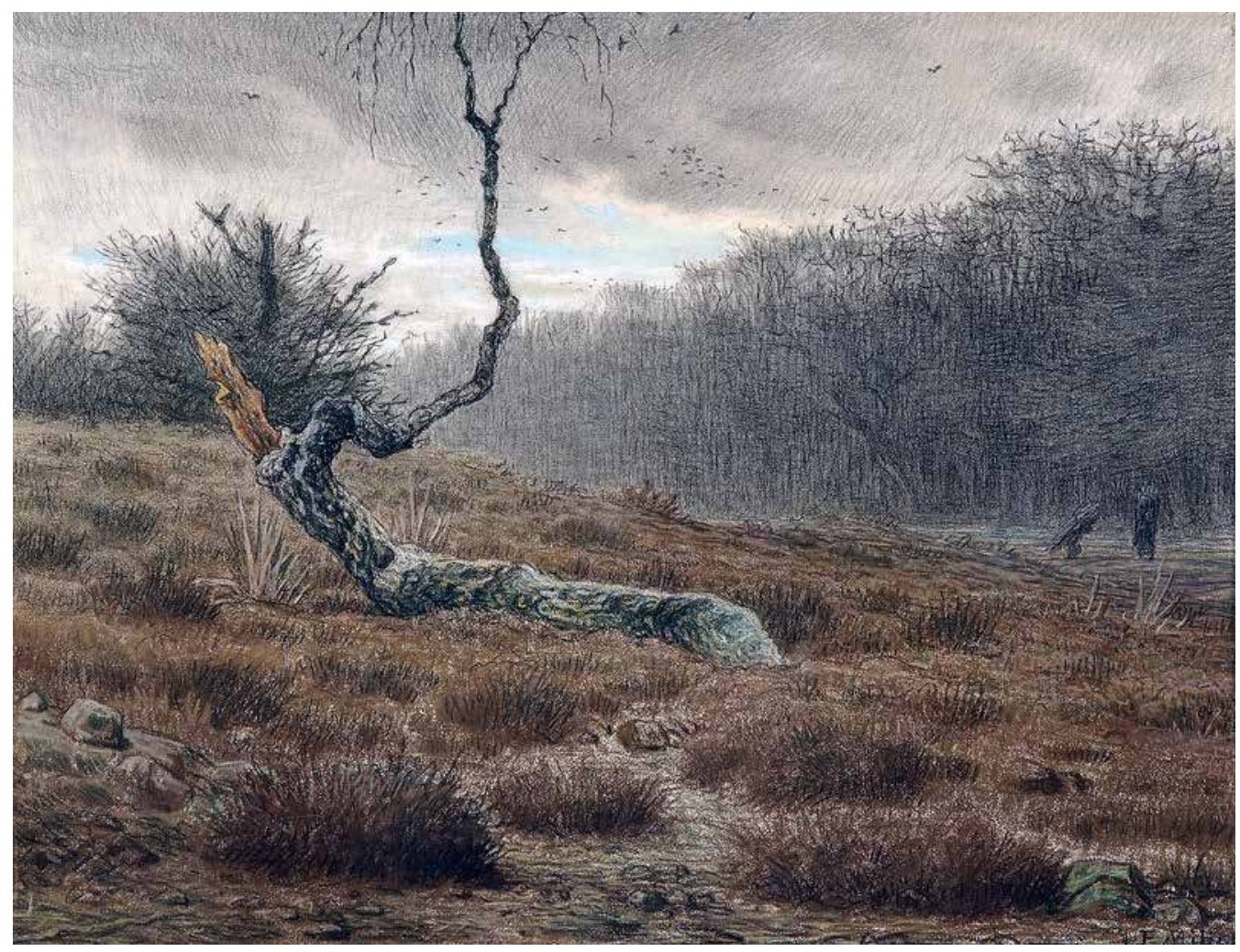

à la création officieuse des premières " séries artistiques " (624 hectares) officialisées par le décret impérial du 13 août 1861, qui instituait la " réserve artistique " : 1097 hectares de la forêt (outre les rochers improductifs) exemptés des coupes réglementaires, " conservés pour les artistes et touristes qui visitent chaque année ce musée d'arbres gigantesques, de sites sauvages, mine inépuisable de modèles pour les paysagistes, de promenades charmantes pour les curieux de tous les pays ${ }^{21} "$. La forêt de Fontainebleau fut ainsi le premier site naturel au monde à faire l'objet d'une mesure de protection, quelques années avant la création en 1872 aux États-Unis du Yellowstone National Park. Pourtant, le combat devait vite reprendre. L'administration forestière ayant annoncé qu'elle effectuerait en 1872 les coupes ordinaires qui n'avaient pu avoir lieu en 1870 et 1871 (du fait de la guerre), on assista à une mobilisation massive des artistes, écrivains et hommes politiques. Un Comité de protection artistique de la forêt fut créé, dont le président d’honneur était Jean-François Millet (fig. 12), et reconnu officiellement le 21 mars 1873 . Une requête fut présentée le 30 juillet 1873 au ministre de l'Instruction publique et des Beaux-Arts, suivie d'une pétition adressée le 31 août 1874 au maréchal de Mac-Mahon, président de la République, suppliant que l'on ordonnât de surseoir à la destruction programmée de 13298 chênes, 4828 hêtres et 1720 hectares de taillis. Elle recueillit 218 signatures dont celles de Victor Hugo, George Sand, Jules Michelet, Henri Martin, ou encore Claude Monet ou le baron Taylor. Alexandre Foucher de Careil (1825-1891), préfet et sénateur de Seine-et-Marne, déposa au Sénat, le 3 avril 1876, une proposition de loi visant à augmenter la superficie des réserves artistiques de 1000 hectares. Le débat fit rage qui opposait les partisans de la protection au directeur général des Forêts, lequel s'appliqua à démontrer le manque à gagner lié à ce refus d'abattage qui faisait de "l'École de Fontainebleau " une école plus chère que les écoles de Rome et d'Athènes réunies !
12. Jean-François Millet, Le

Bouleau mort, carrefour de I'Épine, forêt de Fontainebleau, 1866-1867,

Dijon, musée des Beaux-Arts. 
La loi ne fut pas votée mais un décret de 1892, signé par Carnot, augmenta cependant de 400 hectares la réserve artistique et un nouvel aménagement fut homologué en 1904, qui, tout en laissant au commerce des bois sa part légitime, accentuait l'artialisation de la forêt puisqu'il permettait de maintenir sur pied, indéfiniment et partout, même dans les cantons non réservés, les arbres "dont l'existence perpétue la beauté d'un site ». En 1907 naissait la Société des Amis de la forêt dont le but était de la protéger et la défendre des abus possibles de l'administration forestière en particulier. La naissance de cette société s'inscrit dans un vaste mouvement de défense des paysages, assimilés à des monuments historiques, prônée par le poète Jean Lahor, fondateur en 1901 de la Société pour la protection des paysages de France (aujourd'hui Société pour la protection des paysages et de l'esthétique de la France), laquelle entrait en résonance avec les mots fameux de Victor Hugo, adressés en 1872 au Comité de protection de la forêt de Fontainebleau, et publiés le 7 décembre 1872 dans la Renaissance littéraire : "Il faut absolument sauver la forêt de Fontainebleau [...] Dans une telle création de la nature le bucheron est un vandale. Un arbre est un édifice ; une forêt est une cité, et entre toutes les forêts, la forêt de Fontainebleau est un monument. Ce que les siècles ont construit, les hommes ne doivent pas le détruire. "

\section{Un monument de nature entre restauration, protection et écologie}

" Cette forêt est très mal placée, la plus irrégulière du monde, dans icelle est enclavé un grand nombre de grandes et petites places vides et vagues avec beaucoup de monts et puissants rochers, vallons, fondrières et déserts remplis de bruyère, genêts, genévriers, boulins et autres mauvaises natures des bois, les corps des futaies, demi et quart de futaie d'icelle forêt ont fort peu de suite en leur qualité, âge et nature. " Une forêt stérile et de peu d'intérêt (au moins économique), telle était l'opinion des forestiers en 1858, qui dénonçaient l'irresponsabilité de ses protecteurs auto-proclamés dont l'action avait pour effet d'en brider la gestion, la bonne gestion. Cette gestion impliquait à la fois des coupes dans la futaie, refusées au nom de la patrimonialisation des arbres, et de nouvelles plantations, régénératrices du bois et du sous-bois, vilipendées et combattues, parce qu'elles modifiaient le paysage et ses perspectives, tels que les peintres les avaient connus au début du XIX siècle : un paysage vide et sauvage, malheureusement dénaturé par les plantations des années 1830-1843 (771 ha en feuillus, 4864 ha en résineux, 2234 ha de pins sylvestres). La tension qui régnait entre les professionnels de la forêt et le monde de l'art (et plus largement les usagers de la forêt) persiste, tant il est vrai que les visiteurs s'attendent à " trouver la forêt de Fontainebleau dans son originalité et sa spécificité : massive, riche d'une diversité de lieux, d'ambiances et d'images touristiques figées depuis le début du XIX siècle " (FREYTET, 1996), tant également le statut de Fontainebleau est ambigu, aux yeux mêmes des scientifiques. Paul Arnould (dans ARNOULD, CIESLAK, 2004) constate ainsi, avec quelque étonnement, que la forêt de Fontainebleau est sans doute l'une des forêts de plaine les plus « manipulées " par l'homme - dès le XI ${ }^{\mathrm{e}}$ siècle et ses premiers aménagements pour les «plaisirs du roi ", qui s'amplifient au XVII ${ }^{e}$ siècle, et ô combien au XIX siècle par MM. Denecourt et Colinet - et que, pourtant, c'est aussi l'une des forêts qui évoquent le plus « un milieu naturel ", au point qu'elle sert de référence, dans le cadre du MAB (Man and Biosphere ${ }^{22}$ ) pour l'étude de l'écosystème de référence des forêts tempérées de plaine, comme si, au fond, ce massif ne formait qu'une vaste " réserve artistique et biologique ". L'aura historique demeure qui engendre une nostalgie des paysages du passé, et des obligations pour les aménageurs et les gestionnaires de ce paysage. En 1996, le paysagiste Alain Freytet, après avoir fait un inventaire des principaux motifs propres aux paysages de la forêt de Fontainebleau (le bloc de grès, 
la carrière, le sable, la mare, l'arbre singulier, l'arbre mort, l'alignement, le sentier...), proposait de travailler ce qu'il appelait « les ambiances forestières ", en favorisant « le jeu de la lumière ", le " jeu de la profondeur ", le " jeu de la poussée ", le " jeu du sauvage et de l'artifice ». Sa démarche, d'abord esthétique, devait permettre à l'artiste de retrouver effectivement ce qui avait séduit les peintres du XIX ${ }^{e}$ siècle et avait fait de cette forêt un lieu de prédilection pour toute peinture et toute photographie de paysage. Alain Freytet préconisait en outre "d'aménager " neuf sites de la forêt, dont le carrefour de l'Épine, la Mare-aux-Évées, la lisière du Bas-Bréau ou encore le désert d'Apremont, dans le but de retrouver ce qui en faisait un paysage de prédilection du peintre... mais aussi de les

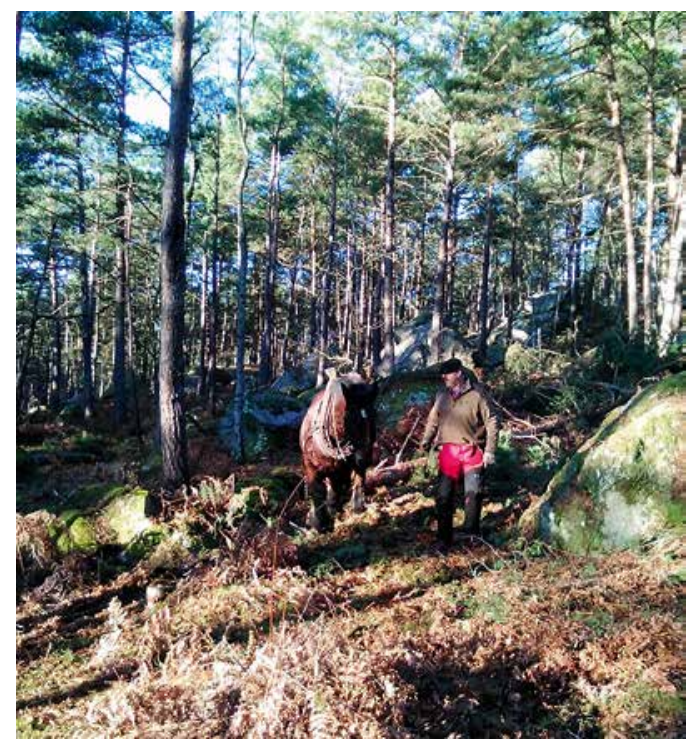
adapter à une fréquentation importante.

S'agissant du désert d'Apremont, il convenait de " retrouver le désert, la clairière, et de dégager les chaos ". Ce travail de " restauration paysagère " est en cours, qui suscite de nombreuses interrogations, auxquelles il est répondu, nous l'avons dit, que " L'ONF met en valeur les paysages des peintres de Barbizon ${ }^{23} "$. Cette assertion apparaît comme le couronnement de la très forte artialisation du lieu, même si Sylvain Ducroux, directeur de projet à l'ONF, et responsable du plan d'aménagement de la forêt, conscient de l'héritage des peintres de Barbizon, mais aussi de "l'état sanitaire médiocre des arbres et de certains sites lié à cet héritage " et des impératifs environnementaux, qui place Fontainebleau aujourd'hui comme hier au cœur des débats forestiers, entend la nuancer. Sans nier ni encore moins renier l'approche patrimoniale, esthétique des travaux en cours, il rappelle aussi qu'en nettoyant ce chaos de ses arbres (fig. 13), il en retrouve et en redessine les contours " naturels ", s'affranchit d'un tracé géométrique mis en place à la fin du XIX ${ }^{e}$ siècle ; $c^{\prime}$ est là une manière de respecter le milieu naturel, tout en restaurant " le sauvage et le vide " présent dans l'inconscient collectif. Une imbrication subtile du naturel et du culturel, de la nature et du monument.
13. Dégagement des chaos rocheux dans la forêt de Fontainebleau par débardage à cheval, travaux réalisés par I'ONF (décembre 2016 - mars 2017), ONF Seine-Nord. 
1. Lettres du 28 juillet 1844, dans la Correspondance d'Eugène Fromentin, Barbara Wright (éd.), Paris, 1995, p. 287 et 288.

2. Barbizon revisited..., 1962-1963; Théodore Rousseau..., 1967-1968 ; L'École de Barbizon..., 1985-1986; Les photographes de Barbizon..., 1991-1992; L'École de Barbizon..., 2002; Unruly Nature..., 2016-2017.

3. Père Dan, Fontainebleau, le Trésor des merveilles de la maison royale, Paris, S. Cramoisy, 1642, p. 17-19.

4. Description historique des chasteau, Bourg et Forêt de Fontainebleau; contenant une explication historique des peintures, tableaux, reliefs, statues, ornements qui s'y voyent et la vie des architectes, peintres et sculpteurs qui y ont travaillé..., À Paris, chez André Cailleau, 1731, 2 vol.

5. "Ces hauteurs arides forment un spectacle curieux à voir pendant l'hiver ; on a sous les yeux un diminutif des Alpes ", dans William Nathaniel Wraxhall, Voyage en France, Paris, J. E. G. Dufour, 1806, II, p. 164.

6. "Ses grès énormes entassés les uns sur les autres, les sombres sapins qui les couronnent, donnent une idée des beaux paysages de la Suisse ", dans Jean-Baptiste Trumet de Fontarce, Impressions de voyage. Italie. 1820, Bar-sur-seine, Vve Saillard, 1897, p. 5.

7. Voir Description géologique des environs de Paris, par MM. G. Cuvier et Alex. Brongniart, Paris, G. Dufour et E. d'Ocagne, 1822.

8. Alexis Donnet, Description des environs de Paris, considérés sous les rapports topographique, historique et monumental, Paris, Treuttel et Würtz, 1824, p. 190.

9. Jules Lacroix de Marlès, Alfred le jeune voyageur en France, Paris, Didier, 1835. p. 367.

10. Henri Taine, Vie et opinions de Monsieur Frédéric Thomas Graindorge, Paris, Hachette, 1867, p. 243.

11. Illusion racontée par Théophile Gautier dans Le Moniteur du 24 août 1860 (article écrit à la suite des obsèques de Decamps auxquelles il a assisté à Fontainebleau).

12. " As-tu vu les sables d'Arbonne? Il y a là un petit Sahara qui doit être gentil à l'heure qu'il est. » Lettre de George Sand à Gustave Flaubert du 31 juillet 1868.

13. F. H. Paillet, Description physique de la forêt de Fontainebleau, Versailles, imprimerie Jacob, 1806, p. 24.
14. J. A. Dulaure, Histoire physique et morale de Paris, depuis les premiers temps historiques jusqu'à nos jours, Paris, Guillaume, 1821, p. 62 .

15. Voir la bibliographie de recueils de paysages lithographiés (1817-1854) dans Jean Adhemar, La France romantique, les lithographies de paysage au XIX $X^{e}$ siècle, Paris, 1997.

16. Notice sur le fossile humain trouvé près Moret département de Seine-et-Marne par M. J. P. Barruel, chez Pinard, 1824.

17. Alfred Sensier, Souvenirs sur Théodore Rousseau, Paris, Léon Techener, 1872 , p. 274.

18. Entre autres : Guide du voyageur dans la forêt de Fontainebleau ou choix de promenades les plus pittoresques, chez l'auteur, 1839 ; Petit indicateur du voyageur à Fontainebleau, ou renseignements indispensables pour séjourner agréablement dans ce pittoresque rendez-vous de plaisance, Paris, aux Librairies du Palais national, 1850 ; Carte-guide $d u$ voyageur à Fontainebleau, chez l'auteur, 1852 ; Itinéraire du palais et de la forêt de Fontainebleau, à l'usage des personnes qui n'ont que peu de jours à consacrer à ce rendezvous de plaisance si pittoresque et si riche de souvenirs, chez l'auteur, 1873...

19. C. F. Denecourt, Ah si l'Empereur le savait!, Paris, imprimerie de Rançon, 1860.

20. Le brouillon de cette lettre est conservé au département des Arts graphiques du musée du Louvre (autographe 3841, L1 85bis). Voir aussi THOMAs, 2000. La lettre est publiée intégralement p. 214-217.

21. Bibliothèque municipale de Fontainebleau, R.C.613.

22. Le Programme sur l'Homme et la biosphère $(\mathrm{MAB})$ est un programme scientifique intergouvernemental visant à établir une base scientifique pour améliorer les relations homme-nature au niveau mondial, lancé au début des années 1970. Voir le site de l'UNESCO : http://www.unesco. org/new/fr/natural-sciences/environment/ ecological-sciences/man-and-biosphereprogramme/ (consulté le 15 juin 2017).

23. ONF, Lettre d'Info, novembre 2016.

\section{Bibliographie sélective}

\section{Ouvrages anciens à caractère de sources}

- AdAM-SAlomon, Asselineau, AudeBRAN, (1855) 2007 : G. Adam-Salomon, C. Asselineau, P. Audebran et al., La Forêt des poètes. Fontainebleau. Hommage à Denecourt, Barbizon, 2007 [éd. orig. : Hommage à C. F. Denecourt, Paris, 1855].

- DoIGNON, 1956-1957 : Pierre Doignon, "Le tourisme à Fontainebleau ", articles publiés dans le journal La Liberté, du 17 juillet 1956 au 29 août 1957.

- DOMET, 1873 : Paul Domet, Histoire de la forêt de Fontainebleau, Paris, 1873.

- Herbet, 1903 : Félix Herbet, Dictionnaire historique et artistique de la forêt de Fontainebleau : routes, carrefours, cantons, gardes, monuments, croix, fontaines, puits, mares, environs, Fontainebleau, 1903 (cet ouvrage est consultable sur le site de la Société des Amis de la forêt de Fontainebleau, www. aaff.org).

- LeVALLOIS, 1875 : Jules Levallois, Fontainebleau. Mémoires d'une forêt, Paris, 1875.

- Michel, 1909 : Émile Michel, La forêt de Fontainebleau dans la nature, dans l'histoire, dans la littérature et dans l'art, Paris, 1909.

\section{Études récentes}

- ARNOUld, CIESLAK, 2004 : Paul Arnould, Caroline Cieslak, " Mise en scène d'objets de nature à Paris et Varsovie : les arbres remarquables de deux forêts périurbaines ", dans Natures Sciences Sociétés, vol. 12, n ${ }^{\circ}$, 2004, p. 157-171, en ligne : http://www. cairn.info/revue-natures-sciences-societes2004-2-page-157.htm (consulté le 15 juin 2017).

- Barbizon revisited..., 1962-1963 : Barbizon revisited, Robert L. Herbert (dir.), cat. exp. (San Francisco, California Palace of the Legion of Honor/Toledo Museum of Art/ Cleveland Museum of Art/Boston, Museum of Fine Arts, 1962-1963), Boston, 1962. - BORIE, 2003 : Jean Borie, Une forêt pour les dimanches. Les Romantiques à Fontainebleau, Paris, 2003.

-BOURDU, JOUSSAUME, VIARD, 1988 : Robert Bourdu, Monique Joussaume, Michel Viard (coll.), Arbres souverains, Paris, 1988. - BRosse, 1989 : Jacques Brosse, Mythologie des arbres, Paris, 1989. 
- Challe, MARbot, 1991 : Daniel Challe, Bernard Marbot, Les Photographes de Barbizon, la forêt de Fontainebleau, Paris, 1991. - CORBIN, 2013 : Alain Corbin, La douceur de l'ombre: l'arbre, source d'émotions, de l'Antiquité à nos jours, Paris, 2013.

- CORvol, 1987 : Andrée Corvol, L'homme au bois : histoire des relations de l'homme et de la forêt du XVII siècle au XXe siècle, Paris, 1987. - Corvol, ARnould, Hotyat, 1997 : Andrée Corvol, Paul Arnould, Micheline Hotyat, La forêt, perceptions et représentations, Paris, 1997.

- L'École de Barbizon..., 1985-1986 : L'École de Barbizon, un dialogue franco-néerlandais, John Sillevis, Hans Kraan (dir.), cat. exp. (Gand, Museum voor schone kunsten/La Haye, Haags gemeentemuseum/Paris, Institut néerlandais, 1985-1986), Madeleine et Raoul Mengarduque (trad. fra.), La Haye/ Paris, 1985.

- L'École de Barbizon..., 2002 : L'École de Barbizon, peindre en plein air avant l'impressionnisme, Vincent Pomarède, Gérard de Wallens, Olivier Meslay et al., cat. exp. (Lyon, musée des Beaux-Arts, 2002), Paris, 2002.

- Environnement, forêt et société..., 2015 : Environnement, forêt tet société $X V I^{e}-X X^{e}$ siècle, actes de la journée d'études (Paris, École nationale supérieure/CNRS, Institut d'histoire moderne et contemporaine, Groupe d'histoire des forêts françaises [GHFF], 2014), , dans Cahier d'études forêt, $\mathrm{n}^{\circ} 25$, 2015.

-FORD, 2016 : Caroline Ford, Natural Interests: The Context over Environment in Modern France, Cambridge, Mass./Londres, 2016.

- La forêt de Fontainebleau..., 2007 : La forêt de Fontainebleau, un atelier grandeur nature, Chantal Georgel (dir.), cat. exp. (Paris, musée d'Orsay, 2007), Paris, 2007.

- FREYTET, 1996 : Alain Freytet, Paysages de la forêt de Fontainebleau. Ambiances, sites et motifs, enjeux et intentions paysagères, Paris, 1996.

- HARRISON, 1994 : Robert Pogue Harrison, Forêts : essai sur l'imaginaire occidental, Paris, 1994.

- Hotyat, 2003 : Micheline Hotyat, "Réserve biologique intégrale du Gros Fouteau en forêt de Fontainebleau : entre dynamiques contrôlées et dynamiques spontanées ", dans Cahiers d'études, Forêts et réserves, $\mathrm{n}^{\circ} 13,2003$, p. 35-43.

-JELOT-BLANC, 2008 : Jean-Jacques JelotBlanc, Si Fontainebleau m'était filmé : Hollywood en forêt, 1908-2008, cent ans de cinéma, Héricy, 2008.
- KALAORA, 1981 : Bernard Kalaora, Le musée vert ou le loisir en forêt. Naissance et développement d'un loisir urbain, le cas de la forêt de Fontainebleau, Paris, 1981.

- KALAORA, 1993 : Bernard Kalaora, Le musée vert, radiographie d'un loisir urbain en forêt de Fontainebleau, Paris, 1993.

- NOTTER, 2007 : Annick Notter, Fontainebleau, son château, sa forêt, l'invention d'un tourisme (1820-1939), Paris, 2007.

- Les photographes de Barbizon..., 1991 1992 : Les photographes de Barbizon, la forêt de Fontainebleau, Daniel Challe, Bernard Marbot, cat. exp. (Paris, BnF, galerie de Photographie, 1991-1992), Paris, 1991.

- Polton, (1985) 1994 : Jean-Claude Polton, Tourisme et nature au XIX $X^{e}$ siècle. Guides et itinéraires de la forêt de Fontainebleau (vers 1820-vers 1880), Paris, 1994 [aux origines de ce livre, fondamental, est une thèse de troisième cycle en histoire contemporaine dirigée par Philippe Vigier et soutenue à l'université de Paris Nanterre en 1985].

- POLTON, 2005 : Jean-Claude Polton, « La forêt de Fontainbleau : aux origines de la protection de la nature en France ", dans La Grappe, $\mathrm{n}^{\circ}$ 63-64, 2005, p. 55-64.

- Polton, 2011 : Jean-Claude Polton, Claude-François Denecourt : 1788-1875, l'amant de la forêt de Fontainebleau?, Fontainebleau, 2011.

- TENDron, 1982 : Gérard Tendron, La Forêt de Fontainebleau, de l'écologie à la sylviculture, Paris, 1982

- Testu, Hermeline, Rey, 1998 : Anne Testut, Michel Hermeline, Geneviève Rey, Lettres de la forêt de Fontainebleau, abécédaire photographique, Paris, 1998.

- Théodore Rousseau..., 1967-1968: Théodore Rousseau 1812-1867, Michel Laclotte, Hélène Toussaint (dir.), cat. exp. (Paris, musée du Louvre, 1967-1968), Paris, 1967.

- Thomas, 2000 : Greg M. Thomas, Art and Ecology in Nineteenth-Century France: the Landscapes of Théodore Rousseau, Princeton, N. J., 2000.

- Unruly Nature..., 2016-2017 : Unruly Nature: The Landscapes of Théodore Rousseau, Scott Allan, Edouard Kopp (dir.), cat. exp. (Los Angeles, The J. P. Getty Museum/ Copenhague, Ny Carlsberg Glyptotek, 2016-2017), Los Angeles, 2016. 